\title{
Circulating Mature Granzyme B+ T Cells Distinguish Crohn's Disease Associated Axial Spondyloarthritis from Axial Spondyloarthritis and Crohn's Disease
}

\section{Adam R. Lefferts}

University of Colorado Anschutz Medical Campus: University of Colorado - Anschutz Medical Campus

\section{Emilie H. Regner}

CU Anschutz: University of Colorado - Anschutz Medical Campus

\section{Andrew Stahly}

CU Anschutz: University of Colorado - Anschutz Medical Campus

\section{Becky O'Rourke}

CU Anschutz: University of Colorado - Anschutz Medical Campus

\section{Mark E. Gerich}

CU Anschutz: University of Colorado - Anschutz Medical Campus

\section{Blair P. Fennimore}

CU Anschutz: University of Colorado - Anschutz Medical Campus

\section{Frank I. Scott}

CU Anschutz: University of Colorado - Anschutz Medical Campus

\section{Alison E. Freeman}

CU Anschutz: University of Colorado - Anschutz Medical Campus

\section{Ken Jones}

CU Anschutz: University of Colorado - Anschutz Medical Campus

Kristine Kuhn ( $\square$ KRISTINE.KUHN@CUANSCHUTZ.EDU)

University of Colorado Anschutz Medical Campus: University of Colorado - Anschutz Medical Campus https://orcid.org/0000-0002-6900-4195

\section{Research article}

Keywords: Crohn's disease, inflammatory bowel disease, axial spondyloarthritis, ankylosing spondylitis, interferonopathy, cytotoxic T cells

Posted Date: January 5th, 2021

DOI: https://doi.org/10.21203/rs.3.rs-138435/v1 
License: (c) (i) This work is licensed under a Creative Commons Attribution 4.0 International License. Read Full License 


\section{Abstract}

Background: Axial spondyloarthritis (axSpA) has strong connections with intestinal inflammation as occurs in Crohn's disease (CD). However, the immunologic mechanisms that distinguish axSpA, CD, and those with features of both diseases (CD-axSpA) are unknown. This study aimed to address this question by initial unbiased single cell RNA-sequencing (scRNAseq) on a pilot cohort followed by validating findings using flow cytometry and ELISA in a larger cohort.

Methods: Two individuals each with CD, axSpA, CD-axSpA, and healthy controls (HC) were recruited for a pilot discovery scRNAseq cohort, and the validation cohort consisted of 18 axSpA, 24 CD, 13 CD-axSpA, and $17 \mathrm{HC}$ that was evaluated by flow cytometry on PBMCs and ELISAs for plasma cytokines.

Results: Uniquely, PBMCs from subjects with CD-axSpA demonstrated a significant increase in granzyme $B+T$ cells of both $C D 4+$ and $C D 8+$ lineages by both scRNAseq and flow cytometry. $T$ cell maturation was also greater in those with CD-axSpA, particularly the $\mathrm{CD} 4+$ granzyme $\mathrm{B}+$ population. Pathway analysis suggested increased interferon response genes in all immune cell populations within CD-axSpA. Although IFN-g was elevated in the plasma of a subset of subjects with CD-axSpA, IL-6 was also significantly elevated.

Conclusions: Our findings support the presence of a chronic interferonopathy in subjects with CD-axSpA characterized by interferon signaling by pathway analysis and an expansion of mature, cytotoxic $T$ cells. These data indicate fundamental immunological differences between CD-axSpA and both of the putative "parent" conditions, suggesting that it is a distinct disease with unique natural history and treatment needs.

\section{Background}

The overlapping conditions of inflammatory bowel disease (IBD) and spondyloarthritis (SpA) present unique challenges in diagnosis and treatment of individuals having both diseases. Seven percent of individuals with Ulcerative Colitis (UC) and 13\% with Crohn's disease (CD) will develop sacroiliitis, and 2\% with UC and $4 \%$ with CD have ankylosing spondylitis (AS) [1]. Conversely, 7\% of patients with axial SpA (axSpA) will develop IBD [2], although many more patients have subclinical bowel inflammation. In spite of this overlap, clinical trials in either IBD or axSpA fail to differentiate patients with overlapping disease. Such a concept is particularly important as highlighted by trials with secukinumab, which is effective in axSpA but worsens CD [3]. While there is increased interest in identifying bowel inflammation in patients with axSpA using biomarkers such as fecal calprotectin, there is a lack of evidence to guide treatment of the overlapping IBD-associated axSpA.

In order to better treat disease, an understanding of pathophysiologic mechanisms is needed. Indeed, many groups have identified unique immune features of axSpA in the circulation, notably by IL-17producing cell populations such as type 3 innate lymphoid cells (ILC3s) [4], mucosal-associated invariant

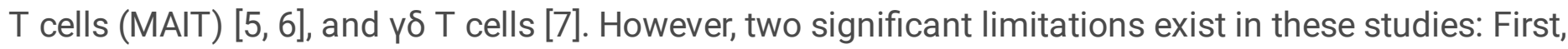


studies were based off flow cytometric analyses searching for specific cell populations; an unbiased approach for identifying altered immune phenotypes has not been performed. Second, axSpA and CD were compared to controls, lacking analysis from those with the overlapping phenotype of IBDassociated axSpA, which may demonstrate unique immune phenotypes.

To begin to address this knowledge gap, we propose an unbiased immune cell profiling using patients with IBD, axSpA, and overlapping conditions that are evaluated and compared to one another using strict definitions. In this report, we attempt such an analysis by comparing immune phenotypes in individuals with CD with ileal involvement, individuals with axSpA meeting ASAS criteria[8] with the additional requirement of positive imaging, and individuals who met the definitions of both CD and axSpA. Using these strict definitions, we began with an unbiased immune phenotyping of peripheral blood in a subset of participants. From those pilot data, we validated findings in the full cohort of recruited subjects. We find that CD-axSpA subjects, compared to $C D$ or axSpA alone, demonstrate a change towards a Th1-like immune response with cytotoxic $T$ cells and an interferon signature, as well as evidence of accelerated $T$ cell maturation.

\section{Methods}

\section{Subject Recruitment}

Utilizing a case-control format, patient and control study subjects were recruited at the University of Colorado Hospital between November 2017 and November 2018. Demographic and clinical data summarizing the study groups are presented in Supp. Table 1. Subjects were identified from the endoscopy schedule if undergoing a routine colonoscopy as part of their clinical care or recruited to undergo an elective flexible sigmoidoscopy. Recruited healthy controls $(n=17)$ were undergoing colonoscopy for routine cancer screening or a change in bowel habits. Subjects with $C D(n=25)$ were undergoing colonoscopies for disease activity assessment and colon cancer/dysplasia screening. Only patients who did not have endoscopic or histologic evidence of dysplasia were recruited into the study. AxSpA cases $(n=20)$ underwent elective flexible sigmoidoscopy for this study or were undergoing colonoscopy due to changes in bowel habits $(n=2)$ and all were evaluated for subclinical bowel inflammation; they were only included as cases when bowel inflammation by histology was excluded. Patients with CD-axSpA similarly underwent either elective flexible sigmoidoscopy $(n=8)$ or standard of care colonoscopy $(n=5)$. Subjects with CD were eligible if they had a diagnosis of such based on evaluation by an IBD-trained gastroenterologist included in the study. Subjects recruited as axSpA cases fulfilled the 2009 Assessment of SpondyloArthritis International Society (ASAS) criteria for axSpA [7], including evidence of axial disease by either MRI or radiographs. Individuals with CD-axSpA met study criteria for both CD and axSpA.

Exclusion criteria for all groups included: presence of bowel disease (except in CD groups in which only CD was allowed), rheumatologic disease (except in the axSpA groups in which only axSpA was allowed), pregnancy, use of antibiotics in the two weeks prior to study entry, cancer or cancer history, inability to 
stop aspirin or non-steroidal anti-inflammatory drugs seven days before and after endoscopy, use of anticoagulation, HIV, and Clostridium difficile infection within the past 3 months.

At the time of endoscopy, subjects completed questionnaires regarding demographic information and disease activity indices including the Harvey Bradshaw Index (HBI) and Bath Ankylosing Spondylitis Disease Activity Index (BASDAI). These data are presented in Supp. Table 1. Blood was collected into tubes containing EDTA for further processing and analysis of cells.

This study was conducted according to the principles within the Declaration of Helsinki. All study procedures were approved by the Colorado Multiple Institutional Review Board. All subjects provided written informed consent.

\section{Processing of PBMCs and Plasma}

Peripheral blood mononuclear cells (PBMCs) were isolated from collected whole blood using gradient centrifugation. Whole blood was diluted with $20 \mathrm{ml}$ sterile PBS, under which $10 \mathrm{ml}$ of Ficoll-Paque PLUS (GE Healthcare) was layered. After centrifugation at 300 RCF for 20 minutes, the buffy coat containing PBMCs was removed. PBMCs were washed with sterile PBS, counted, and cryogenically stored at a concentration of $1 \times 10^{6} \mathrm{cells} / \mathrm{ml}$ in recovery freezing media (Gibco) until use.

Whole blood collected in a second K2-EDTA tube was centrifuged at $2000 \mathrm{~g}$ for 5 minutes, and the plasma was removed and stored at $-80^{\circ} \mathrm{C}$. Plasma cytokines were evaluated using a multiplex platebased platform for the cytokines IFN-a2a (lower limit of detection, LLOD $=4.0 \mathrm{pg} / \mathrm{ml}$ ), IFN- $\beta$ (LLOD = $3.1 \mathrm{pg} / \mathrm{ml}), \mathrm{IFN}-\gamma(\mathrm{LLOD}=1.7 \mathrm{pg} / \mathrm{ml}), \mathrm{IL}-6(\mathrm{LLOD}=0.33 \mathrm{pg} / \mathrm{ml}), \mathrm{IL}-23(\mathrm{LLOD}=1.4 \mathrm{pg} / \mathrm{ml})$, and combined IL-17A/F (LLOD = $1.8 \mathrm{pg} / \mathrm{ml}$ ) (Meso Scale Discovery).

\section{Single cell RNA sequencing}

Cells were stained using anti-human CD45 PE (clone H130, Biolegend) and viability dye (Ghost Dye 510, Tonbo). Viable CD45 + cells were sorted on a Moflo Astrios EQ by the flow cytometry core at the University of Colorado Cancer Center. Target cell number was 5,000 to 10,000 cells. Cells were submitted to the Genomics Core for sequencing using the $10 x$ Platform (10x genomics). Recovered cell number and sequencing depth for each sample is reflected in Supp. Table 2. Sequence data was then analyzed as follows: Cellranger (2.0.2) count module was used for alignment, filtering, barcode counting and UMI counting of the single cell FASTQs. 2D UMAP plots and clustering were determined by the following method: Seurat (V3) was used to filter cells to include only those with $>200$ and $<4000$ genes, mitochondrial gene expression $<10 \%$, and total counts $<20000$. The Seurat module IntergrateData with $\operatorname{dims}=1: 20$ was used to integrate the blood and colon SCRNAseq data from all 8 samples. FindClusters with resolution $=0.25$ on PCA reduced data using 30 principal components was used to define clusters. UMAP coordinates were determined by Seurat. Pathway enrichment scores were generated through the use of IPA (QIAGEN Inc.).

\section{Flow cytometry}


Cells were stained with the indicated antibody cocktail (Supp. Tables 3-4) for 30 minutes in PBS (Gibco) with $10 \%$ FBS (Sigma) at $4{ }^{\circ} \mathrm{C}$. Following staining, cells were pelleted at $500 \mathrm{~g}$ for 10 minutes and washed 3 times in $2 \mathrm{ml} \mathrm{PBS}$. Cells were fixed for 20 minutes (Foxp3 Transcription factor Staining kit, Tonbo).

Samples were additionally stained for 30 minutes for intracellular markers before analysis. Samples were run on a 5-laser Becton Dickinson LSR Fortessa X-20. Analysis was carried out in FloJo V10 by Treestar. Samples with $<60 \%$ viability were excluded from analysis (mean $78.8 \%$ ).

\section{Data Analysis}

Data were evaluated for normality using D 'Agostino test. Normal data were evaluated by ANOVA followed by t-test with Welch's correction and non-parametric data by Mann-Whitney. Statistical analyses and graphics were conducted with GraphPad 8.2 and Microsoft Excel Office Professional Plus 2013.

\section{Results}

Single cell RNA sequencing indicates mature, cytotoxic T cells and an interferon activation signature distignuish $C D$-axSpA from axSpA and $C D$

To understand what cellular populations distinguish CD, axSpA, and co-morbid CD-axSpA we first used an unbiased approach by performing high-depth single-cell RNA sequencing (scRNAseq) of the peripheral blood on a pilot cohort (Supplementary Table 2). Due to the limited sample number in this application, we kept the 8 subjects as similar as possible, choosing all men, with age, disease activity, and medication use matching the larger study group. 2D UMAP analysis from the pilot cohort did not identify a uniquely expanded cell type in any disease state (Supp. Figure 1). We did, however, observe an increase in granzyme $b(G Z M B)$ expression within memory T cells in all disease states relative to HCs, with the highest expression in CD-axSpA (Fig. 1a-c). We additionally noted increased expression of the innate-like transcription factor promyelocytic leukemia zinc finger ( $P L Z F)$ (Fig. 1d-f) within the memory CD4 compartment in both $\mathrm{CD}$ and axSpA, but not CD-axSpA, suggesting that some of the memory CD4 compartment in CD and axSpA comprised PLZF expressing innate-like T cells such as $\gamma \delta T$ cells. Finally, we utilized Ingenuity Pathway Analysis (IPA) to evaluate unique activation signatures in each UMAP cellular cluster and observed an interferon signature across all cell types in CD-axSpA (Fig. 1g), and the absence of such a signature in both $\mathrm{CD}$ and axSpA (Supp. Figure 2).

\section{Mature GZMB + T cells are expanded in the circulation of subjects with CD-axSpA}

The increased GZMB expression in memory T cells in our scRNAseq data and the IPA analysis with a pervasive interferon response led us to probe the circulating $T$ cell populations for the presence of cytotoxic T cells. (Consistent with the single cell data, there were no gross differences in B cells, plasma cells, pDCs, or CD11c + mononuclear phagocytes in the peripheral blood (Supp. Figure 3a-d).) We observed a small increase in GZMB + T cells of both CD4 (Fig. 2a) and CD8 (Fig. 2b) lineages in CDaxSpA. Within these GZMB + subsets we also observed an increase in the NKG2a + population within the 
GZMB + sub-populations from the CD-axSpA subjects (Fig. 2c, d), which is potentially indicative of a chronically activated state [9]. We next assayed for the presence of cell surface Lamp1, a marker indicative of the recent release of cytotoxic granules [10], but did not observe significant increase in cell surface Lamp1 on CD4 + GZMB + or CD8 + GZMB + T cells in CD-axSpA (Sup Fig. 4a-b), suggesting that if these cells are indeed de-granulating, they are not doing so in the peripheral blood. $\gamma \delta T C R+T$ cells were significantly expanded in the PBMCs of patients with $C D$ compared to $\mathrm{HC}$, and there was a trend towards an increase in axSpA $(P=0.057)$; however, there was no increase in subjects with $C D$-axSpA compared to $\mathrm{HC}$ (Fig. 2e) nor was GZMB expression increased on $\gamma \delta \mathrm{T}$ cells in any group (Fig. 2f). These findings suggest that both CD4 + and CD8 + cytotoxic T cells may contribute to the pathogenesis of CD-axSpA, while $\gamma \delta T$ cells, which have been linked to both $C D$ and $A S[7,11]$, may not be as significant in CD-axSpA.

The observations of increased cytotoxic T cells and interferon signaling pathway activation led us to question if the CD-axSpA patients also exhibited altered T cell maturation. PBMCs from patients with CDaxSpA had a marked decrease in circulating naïve CD45RA + CCR7 + CD4 + T cells (Fig. 3a) as well as an increase in CD45RA- CCR7- effector memory (EM) CD4 + T cells (Fig. 3b), particularly GZMB $+T_{E M}$ (Fig. 3c), but not CD45RA- CCR7 + central memory (CM) T cells (Supp. Figure 5a). There was a small decrease in naïve $C D 8+T$ cells as well (Fig. $3 d$ ), but without increases in memory CD $8+T$ cell subsets (Supp. Figure $5 b, c$ ). While the CD $4+$ compartment within CD-axSpA was skewed towards a terminally activated phenotype, we noted a small trend towards increased PD- $1+T$ cells of both CD4 and CD8 lineages within all disease groups including CD-axSpA (Fig. 3e, f), suggesting increased terminally exhausted $T$ cells in at leas a subset of individuals.

\section{CD-axSpA is distinguished from axSpA by elevated circulating IFN-y and IL-6}

Due to the presence of an interferon signaling profile suggested by our scRNAseq IPA analysis of the peripheral blood in $\mathrm{CD}$-axSpA, as well as an expansion of circulating mature cytotoxic $T$ cells in these subjects, we assessed plasma interferons. Since we were unable to conclusively determine from IPA alone if the signaling profile was the result of type 1 (IFN- $\alpha 2 a$ and IFN- $\beta$ ) or type 2 interferons (IFN- $\gamma$ ), we chose to examine all three. Type 3 interferons do not signal in human PBMCS [12]; therefore, they were not included. We additionally assayed for Th17 pathway cytokines IL-6, IL-17A/F, and IL-23 given their relevance to $C D$ and $A S$. IFN-y was significantly increased in the plasma of patients with $C D$ but only in a portion of those with CD-axSpA compared to controls (Fig. 4a), suggesting that some individuals have elevated circulating IFN- $\gamma$ but other individuals with CD-axSpA derive their cellular IFN signature from signals elsewhere. There were no significant elevations in type 1 interferons in the disease groups (Supp. Figures $6 a, b)$. We observed a significant increase in IL-6 levels in CD-axSpA relative to the other groups (Fig. 4b). IL-17A/F and IL-23 levels did not differ significantly among the groups (Supp. Figures 6c, d). These data suggest that the increased interferon transcriptional signature in CD-axSpA may be due to increased circulating INF-y in only a subset of individuals, but given that IL- 6 can synergize with interferons to potentiate interferon responses $[13,14]$, IL- 6 also may be a key factor in driving the interferon signature in CD-axSpA. 


\section{Discussion}

While the clinical connection between IBD and SpA has long been appreciated, there are a lack of studies that directly compare the diseases to determine the immunologic relationship these conditions have with one another. Here, we present evidence that CD-axSpA is immunologically distinct from CD or axSpA by starting with an unbiased approach of scRNAseq to survey immune phenotypes and followed by validation through flow cytometry and ELISA. Compared to the other groups, subjects with CD-axSpA demonstrated increased circulating cytotoxic T cells of both CD4 and CD8 lineage with an overall skewing of the T cell compartment towards an activated, effector phenotype, and increased plasma IFN-y plus IL-6. These differing features between groups suggest that CD-axSpA may be a distinct disease entity from both $\mathrm{CD}$ and axSpA, potentially arising either as an exacerbated state of $\mathrm{CD}$ or axSpA characterized by new immunopathology or as a distinct disease entity with a unique natural history.

Our study is not the first to indicate cytotoxic T cells or IFN-y in the setting of axSpA. Classically, the linkage between AS and the class I molecule HLA-B27 has suggested a CD8 + T cell pathology. Yet CD8 + $T$ cells are significantly reduced in individuals with AS along with reduced expression of cytotoxic genes granzyme $A(G Z M A)$ and perforin (PRF1), but synovial fluid levels of GZMA and GZMB were significantly elevated compared to the peripheral blood of the same patients. Intriguingly, the differences in cytotoxic gene expression were limited to men with AS $[15,16]$. In our study, while total CD8 + T cells were unchanged in axSpA, we identified a profound shift of cytotoxic cell populations in our study, and in particular, T cells expressing GZMB in the peripheral blood of individuals with CD-axSpA. The specific role of these cells in disease pathology of CD-axSpA versus other disease states, though, remains unclear.

Of curiosity is the novel finding of expanded GZMB + CD4 + TEM cells in our subjects with CD-axSpA. Cytotoxic CD $4+T$ cells have been shown to be potent killers of antigen presenting cells [17], and colonic epithelial cells upregulate antigen presentation in the context of IBD [18], it is possible that the cytotoxic CD4 cells observed in CD-axSpA directly mediate bowel disease through cytolytic killing of epithelial cells. Equally, these cells may have targets within the joint space, and/or influence pathology through the direct proteolytic effects of granular proteins. Therefore, elucidating the potential role of cytotoxic $T$ cells in the joint will require an understanding of the potential cellular targets, with the caveat that they may have little or no meaningful impact in disease pathogenesis, and their generation is simply a side effect of the presence of elevated systemic interferon.

Our findings point towards IFN- $\gamma$ being a key driver of CD-axSpA, since cytotoxic T cells of both CD4 and CD8 lineages respond to IFN-y $[19,20]$, and we observed a profound IFN response gene signature by IPA. The loss of circulating naïve T cells in our subjects with CD-axSpA may also be a consequence of a chronic interferon response, as this has been observed in other conditions characterized by chronic interferon exposure such as Down's syndrome and hepatitis $C$ infection [21,22]. Our study, though, does not address the source(s) of the elevated IFN-y and IL-6 observed in CD-axSpA, and it remains unclear whether these cytokines are primary drivers of, or a consequence following from, the underlying pathology. 
Our data are in partial agreement with Smith et al. who previously identified increased IFN-y responses in ex vivo derived macrophages from circulating monocytes in individuals with AS [23]. The derived macrophages from AS versus healthy controls demonstrated an elevated IFN- $\gamma$ responsive gene signature although they expressed less IFN- $\gamma$; however, when stimulated with IFN- $\gamma$, the derived macrophages did not upregulate IFN- $\gamma$ response genes like healthy controls, suggesting an intrinsic defect in the IFN- $\gamma$ pathway in patients with AS [23]. We did not observe the same IFN-y response in monocytes from axSpA subjects, but rather in our CD-axSpA subjects, in our scRNAseq dataset. Such discrepancies may be due to limited numbers in our scRNAseq analysis, our more restrictive subsetting of axSpA with and without concomitant CD, or the effect of ex vivo differentiation in the Smith et al. study [23].

IFN-y also has been linked to IBD primarily acting through weakening of epithelial junctions in the intestine [24]. Thus, it seems likely that IFN-y acts as a driver of the intestinal pathology observed in CDaxSpA. IL-6 has also been linked to disease activity in IBD [25]. Furthermore, IFN-y may be involved in the joint disease observed in CD-axSpA, as it has the potential to disrupt osteoclast differentiation through suppression of the RANK signaling pathway [26]. IL-6, by a distinct mechanism involving suppression of the NF-KB and JNK pathways, is also known to suppress osteoclast differentiation [27]. As such, IFN- $\gamma$ and IL-6 may be able to mediate joint damage through different mechanisms than the classically IL-17driven processes observed in axSpA [28]. Additionally, the synergistic effects of concurrent IL-6 and IFN-y signaling $[13,14]$, which amplifies IFN-y responses through IL-6 mediated IRF1 and IRF9 expression, may contribute to the unique pathology observed in CD-axSpA and explain why there was an interferon response signature in $\mathrm{CD}$-axSpA and not CD, despite similar plasma IFN-y levels.

Unfortunately, this study is underpowered to determine the answers to a number of additional critical questions regarding CD-axSpA. For example, distinctions between B27 + and B27- presentations of both axSpA and CD-axSpA may be missed as we included only B27 + individuals in our discovery cohort. Having a larger discovery cohort would have allowed for the discernment of more subtle phenotypes. Additionally, usage of TNF inhibitors within our study cohort makes comparisons between each disease state and healthy controls more difficult. We chose to utilize patients under treatment due to the lack of new diagnosis CD-axSpA patients, as in our cohort patients were invariably under treatment for either CD or axSpA by the time of a diagnosis of CD-axSpA. However, our cohort is well matched for TNF inhibitor usage between disease states, making internal comparisons within diseases important as each group responds differently to treatment, highlighting underlying immunological differences between $C D, a x S p A$, and CD-axSpA. Ideally, patient samples would be collected at the time of diagnosis to remove the confounding effects of treatment, however due to the unique features of CD-axSpA, a prospective cohort will be required to more fully explore the immunological basis of this disease.

\section{Conclusions}

This study provides evidence, by cytokine signature and cellular immunophenotype, that CD-axSpA may be best thought of as a distinct disease entity, instead of an exaggerated form of either CD or axSpA. We caution that the data are limited to a highly defined clinical phenotype and may not explain other forms 
of IBD or peripheral SpA in conjunction with IBD. Yet our findings highlight the need for studies in wellphenotyped populations in order to understand the constellation of diseases under the SpA and IBD umbrellas that are likely immunologically distinct entities. Such studies can then shed light on how best to treat these complex patients.

\section{Abbreviations}

IBD, inflammatory bowel disease

$\mathrm{CD}$, Crohn's disease

UC, ulcerative colitis

AS, ankylosing spondylitis

SpA, spondyloarthritis

axSpA, axial spondyloarthritis

CD-axSpA, Crohn's disease associated axial spondyloarthritis

scRNAseq, single cell RNA sequencing

PBMC, peripheral blood mononuclear cells

ASAS, Assessment of SpondyloArthritis International Society

BASDAl, Bath Ankylosing Spondylitis Disease Activity Index

HBI, Harvey Bradshaw Index

GZMB, granzyme b

$P L Z F$, innate-like transcription factor promyelocytic leukemia zinc finger

Th1, type 1 helper T cells

Th17, type 17 helper T cells

TEM, effector memory T cells

TCM, central memory $\mathrm{T}$ cells

\section{Declarations}

Ethical Approval and Consent to Participate 
This study was conducted according to the principles within the Declaration of Helsinki. All study procedures were approved by the Colorado Multiple Institutional Review Board (protocol \#14-1507). All subjects provided written informed consent and authorization for release of personal health information. An independent safety officer (Lisa Davis, MD) was assigned and met annually with the investigators to conduct safety reviews.

Consent for Publication

Not applicable.

\section{Availability of Data and Materials}

All data are available upon request. Sequencing data is publicly available in the Gene Expression Omnibus (GEO) repository under accession GSE163314.

\section{Competing Interests}

Our work was supported, in part, through a Global Medical Grant by Pfizer awarded to KAK. However, Pfizer had no role in the design of the study; collection, analysis, and interpretation of the data; or writing of the manuscript.

\section{Funding}

ARL and EHR were supported by NIH award T32 AR007534. Additional support was provided by U01 Al101990 sub-award, Boettcher Foundation Webb-Waring Biomedical Research Award, Bruckel Early Investigator Award in AxSpa from the Spondylitis Association of America, and a Pfizer Global Medical Grant.

\section{Authors' Contributions}

$A R L, E H R, K J$, and KAK designed the study. EHR, MEG, BPF, FIS, and AEF recruited subjects and collected tissues. EHR, ARL, and AS processed tissues and generated data. ARL, AS, BO, KJ, and KAK analyzed and interpreted the data. Initial drafts of the manuscript were written by ARL and KAK. All authors contributed to reviewing and revising the manuscript and approved its final form for submission.

\section{Acknowledgements}

The authors would like to thank Lisa Davis, MD, for her participation in ensuring the safety of patients during the study.

\section{Authors' Information}

All work for the study was performed at the University of Colorado Anschutz Medical Campus. Authors AEF and KJ have changed institutional affiliations to Cascade Gastroenterology, Bend, OR (AEF), and 
Department of Cell Biology, University of Oklahoma Health Sciences Center, Oklahoma City, OK (KJ) at the time of manuscript submission.

\section{References}

1. Karreman MC, Luime JJ, Hazes JMW, Weel AEAM. The Prevalence and Incidence of Axial and Peripheral Spondyloarthritis in Inflammatory Bowel Disease: A Systematic Review and Metaanalysis. Journal of Crohn's and Colitis 2016:jjw199.

2. Stolwijk C, Van Tubergen A, Castillo-Ortiz JD, Boonen A: Prevalence of extra-articular manifestations in patients with ankylosing spondylitis: a systematic review and meta-analysis. Annals of the Rheumatic Diseases 2015, 74(1):65-73.

3. Hueber W, Sands BE, Lewitzky S, Vandemeulebroecke M, Reinisch W, Higgins PDR, Wehkamp J, Feagan BG, Yao MD, Karczewski M, et al. Secukinumab, a human anti-IL-17A monoclonal antibody, for moderate to severe Crohn's disease: unexpected results of a randomised, double-blind placebocontrolled trial. Gut. 2012;61(12):1693-700.

4. Ciccia F, Guggino G, Rizzo A, Saieva L, Peralta S, Giardina A, Cannizzaro A, Sireci G, De Leo G, Alessandro R, et al. Type 3 innate lymphoid cells producing IL-17 and IL-22 are expanded in the gut, in the peripheral blood, synovial fluid and bone marrow of patients with ankylosing spondylitis. Ann Rheum Dis. 2015;74(9):1739-47.

5. Toussirot E, Laheurte C, Gaugler B, Gabriel D, Saas P. Increased IL-22- and IL-17A-Producing MucosalAssociated Invariant T Cells in the Peripheral Blood of Patients With Ankylosing Spondylitis. Front Immunol. 2018;9:1610.

6. Gracey E, Qaiyum Z, Almaghlouth I, Lawson D, Karki S, Avvaru N, Zhang Z, Yao Y, Ranganathan V, Baglaenko Y, et al. IL-7 primes IL-17 in mucosal-associated invariant T (MAIT) cells, which contribute to the Th17-axis in ankylosing spondylitis. Ann Rheum Dis. 2016;75(12):2124-32.

7. Kenna TJ, Davidson SI, Duan R, Bradbury LA, McFarlane J, Smith M, Weedon H, Street S, Thomas R, Thomas GP, et al. Enrichment of circulating interleukin-17-secreting interleukin-23 receptor-positive gamma/delta T cells in patients with active ankylosing spondylitis. Arthritis Rheum. 2012;64(5):1420-9.

8. Rudwaleit M, Van Der Heijde D, Landewe R, Listing J, Akkoc N, Brandt J, Braun J, Chou CT, CollantesEstevez E, Dougados M, et al. The development of Assessment of SpondyloArthritis international Society classification criteria for axial spondyloarthritis (part II): validation and final selection. Ann Rheum Dis. 2009;68(6):777-83.

9. Graham CM, Christensen JR, Thomas DB. Differential induction of CD94 and NKG2 in CD4 helper T cells. A consequence of influenza virus infection and interferon-?? 2007, 121(2):238-247.

10. Shen DT, Ma JS, Mather J, Vukmanovic S, Radoja S. Activation of primary T lymphocytes results in lysosome development and polarized granule exocytosis in CD 4 + and CD 8 + subsets, whereas expression of lytic molecules confers cytotoxicity to CD8 + T cells. J Leukoc Biol. 2006;80(4):82737. 
11. Catalan-Serra I, Sandvik AK, Bruland T, Andreu-Ballester JC. Gammadelta T Cells in Crohn's Disease: A New Player in the Disease Pathogenesis? J Crohns Colitis. 2017;11(9):1135-45.

12. Dickensheets H, Sheikh F, Park O, Gao B, Donnelly RP. Interferon-lambda (IFN-) induces signal transduction and gene expression in human hepatocytes, but not in lymphocytes or monocytes. $\mathrm{J}$ Leukoc Biol. 2013;93:377-85.

13. Erb HH, Langlechner RV, Moser PL, Handle F, Casneuf T, Verstraeten K, Schlick B, Schafer G, Hall B, Sasser K, et al: IL6 sensitizes prostate cancer to the antiproliferative effect of IFNalpha2 through IRF9. Endocr Relat Cancer 2013, 20(5):677-689.

14. Cauvi DM, Cauvi G, Toomey CB, Jacquinet E, Pollard KM. From the Cover: Interplay Between IFNgamma and IL-6 Impacts the Inflammatory Response and Expression of Interferon-Regulated Genes in Environmental-Induced Autoimmunity. Toxicol Sci. 2017;158(1):227-39.

15. Gracey E, Yao Y, Qaiyum Z, Lim M, Tang M, Inman RD. Altered Cytotoxicity Profile of CD8 + T Cells in Ankylosing Spondylitis. Arthritis Rheumatol. 2020;72(3):428-34.

16. Gracey E, Yao Y, Green B, Qaiyum Z, Baglaenko Y, Lin A, Anton A, Ayearst R, Yip P, Inman RD. Sexual Dimorphism in the Th17 Signature of Ankylosing Spondylitis. Arthritis Rheumatol. 2016;68(3):67989.

17. Grogg D, Hahn S, Erb P. CD4 + T cell-mediated killing of major histocompatibility complex class IIpositive antigen-presenting cells (APC) III. CD4 + cytotoxic T cells induce apoptosis of APC. 1992, 22(1):267-272.

18. Dotan I, Allez M, Nakazawa A, Brimnes J, Schulder-Katz M, Mayer L. Intestinal epithelial cells from inflammatory bowel disease patients preferentially stimulate CD4 + T cells to proliferate and secrete interferon. 2007, 292(6):G1630-G1640.

19. Tau GZ, Cowan SN, Weisburg J, Braunstein NS, Rothman PB. : Regulation of IFN-y Signaling Is Essential for the Cytotoxic Activity of CD8 + T Cells. J Immunol. 2001;167:5574-82.

20. Brown DM, Lee S, Garcia-Hernandez Mde L, Swain SL. Multifunctional CD4 cells expressing gamma interferon and perforin mediate protection against lethal influenza virus infection. J Virol. 2012;86(12):6792-803.

21. Bloemers BLP, Bont L, De Weger RA, Otto SA, Borghans JA, Tesselaar K. Decreased Thymic Output Accounts for Decreased Naive T Cell Numbers in Children with Down Syndrome. 2011, 186(7):45004507.

22. Sheiko MA, Golden-Mason L, Giugliano S, Hurtado CW, Mack CL, Narkewicz MR, Rosen HR. CD4 + and CD8 + T Cell Activation in Children with Hepatitis C. 2016, 170:142-148.e141.

23. Smith JA, Barnes MD, Hong D, DeLay ML, Inman RD, Colbert RA. Gene expression analysis of macrophages derived from ankylosing spondylitis patients reveals interferon-gamma dysregulation. Arthritis Rheum. 2008;58(6):1640-9.

24. Langer V, Vivi E, Regensburger D, Winkler TH, Waldner MJ, Rath T, Schmid B, Skottke L, Lee S, Jeon $\mathrm{NL}$, et al: IFN-y drives inflammatory bowel disease pathogenesis through VE-cadherin-directed vascular barrier disruption. Journal of Clinical Investigation 2019. 
25. Waldner MJ, Neurath MF. Master regulator of intestinal disease: IL-6 in chronic inflammation and cancer development. Semin Immunol. 2014;26(1):75-9.

26. Ji JD, Park-Min KH, Shen Z, Fajardo RJ, Goldring SR, McHugh KP, Ivashkiv LB. Inhibition of RANK Expression and Osteoclastogenesis by TLRs and IFN- in Human Osteoclast Precursors. 2009, 183(11):7223-7233.

27. Yoshitake F, Itoh S, Narita H, Ishihara K, Ebisu S. Interleukin-6 Directly Inhibits Osteoclast Differentiation by Suppressing Receptor Activator of NF-KB Signaling Pathways. 2008, 283(17):11535-11540.

28. Raychaudhuri SP, Raychaudhuri SK. Mechanistic rationales for targeting interleukin-17A in spondyloarthritis. 2017, 19(1).

\section{Figures}



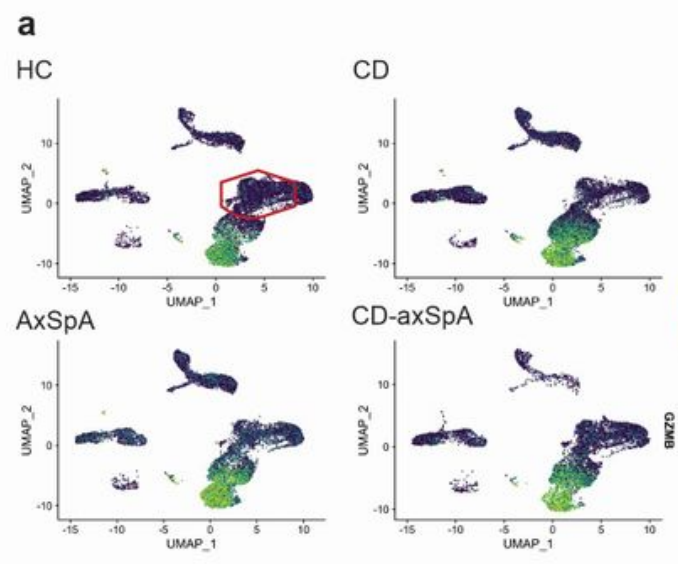

b

c
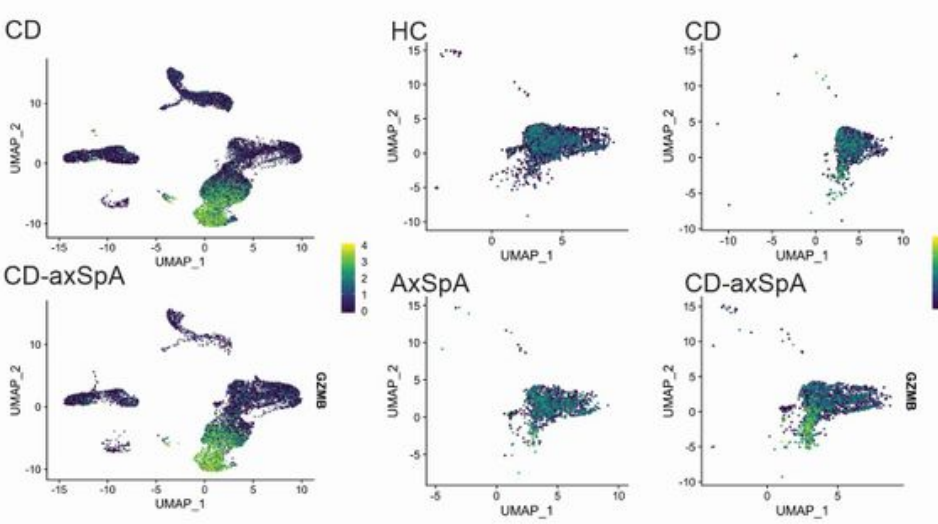

Granzyme B expression in Memory CD4
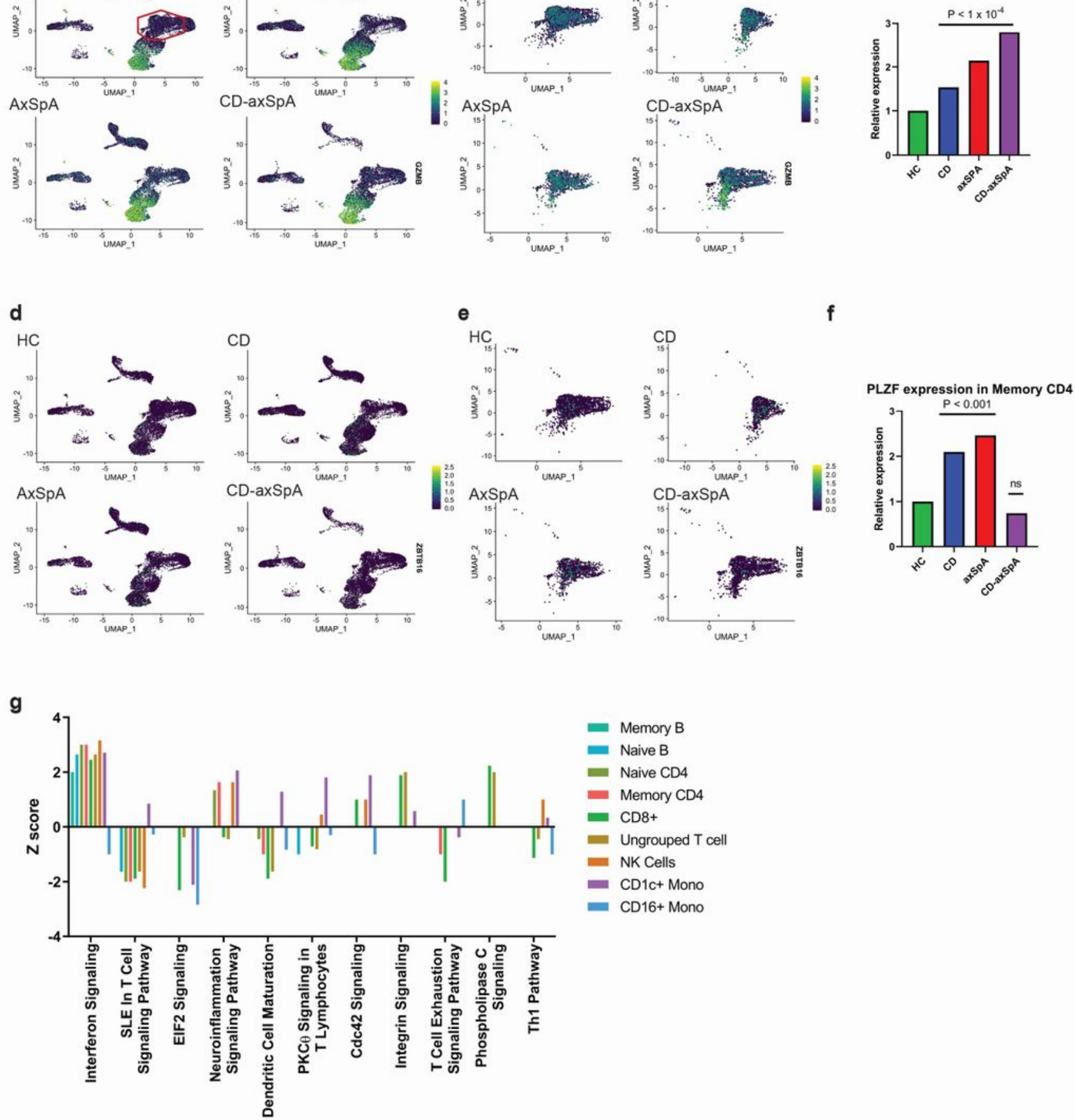

\section{Figure 1}

scRNAseq of PBMCs suggests an expansion of cytotoxic T cells in all disease states as well as an interferon response signature in CD-axSpA. Viable CD45+ cells sorted from PBMCs collected from 2 subjects in each group, HC, CD, axSpA, and CD-axSpA underwent scRNAseq. UMAP with (a, d) all cells, (b, e) within the memory $T$ cell population and $(c, f)$ relative transcript level demonstrate $(a-c)$ granzyme $B$ and (d-f) PLZF expression in memory T cells across the subject groups. Intensity of expression is shown 
as blue (low) to moderate (green) to high (yellow) as indicated by the figure legends. The red gate in (a) identifies the approximate UMAP coordinates corresponding to the memory $T$ cell population represented in (b, e). Significance as shown on the graphs was determined by Wilcoxon rank-sum test. (g) Pathway analysis of the PBMC cellular clusters from the 2 subjects with CD-axSpA was performed, and the Zscores for pathways with at least 1 cell type with an absolute Z-score $\geq 1$ is shown.

a

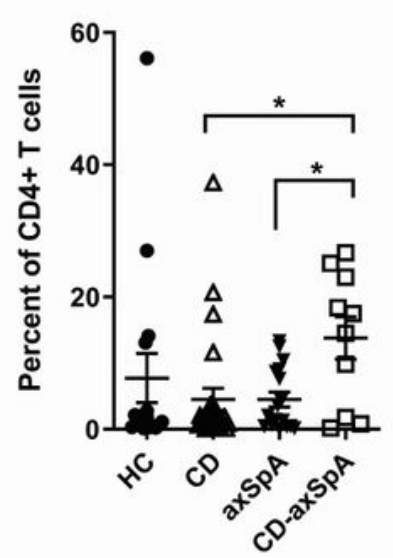

C

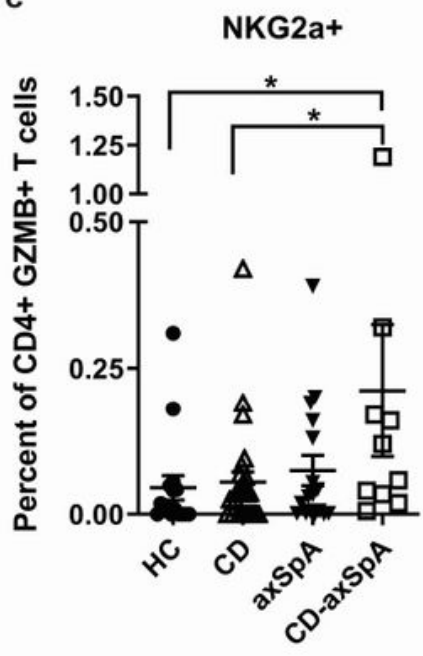

e

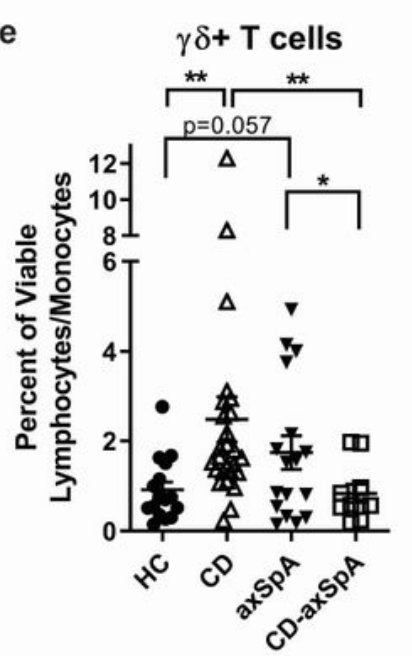

b

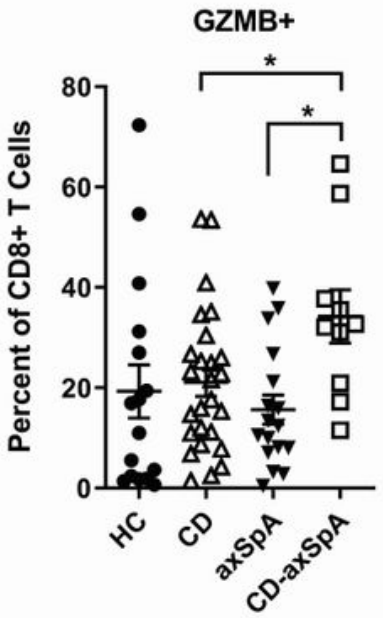

d

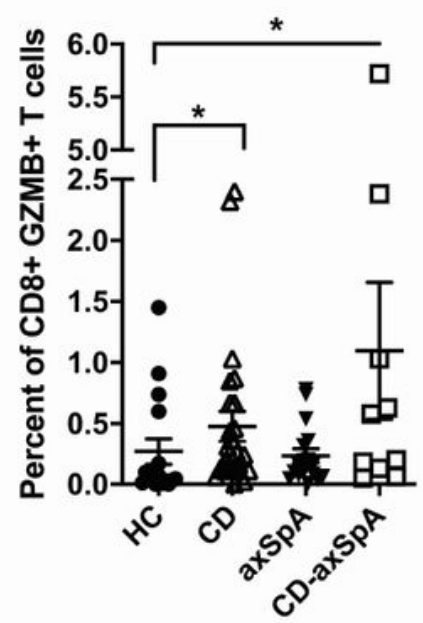

GZMB+

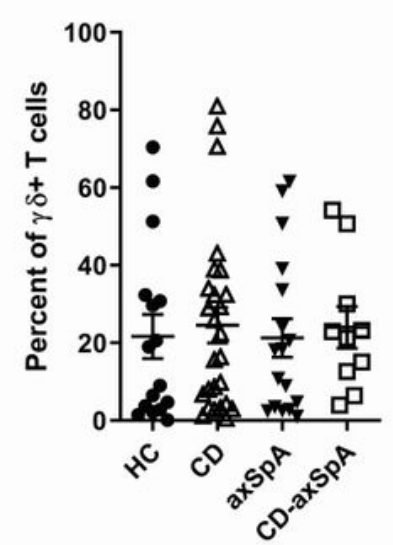

Figure 2 
Cytotoxic GZMB+ T cells are expanded in CD-axSpA. Singlet, viable lymphocytes were gated for CD3+ $\mathrm{TCR} \otimes+$ followed by CD4+ and CD8+ subsets. (a) Within the CD4+ and (b) CD8+ T cell populations, the percent GZMB+ are shown. Next, within the (c) CD4+ GZMB+ and (d) CD8+ GZMB+ T cell subpopulations, the percent expressing the inhibitory co-receptor NKG2a+ are shown. (e) Within the viable lymphocyte gate, the percent TCRQD+ and within that population (f) the percent GZMB+ are shown. All data are individual subject values (symbols) with bars as the mean \pm SEM. ${ }^{*}, \mathrm{P}<0.05 ;{ }^{*}, \mathrm{P}<0.01$ as determined by t-test for parametric data and Mann-Whitney for non-parametric data as determined by D'Agostino test.

a

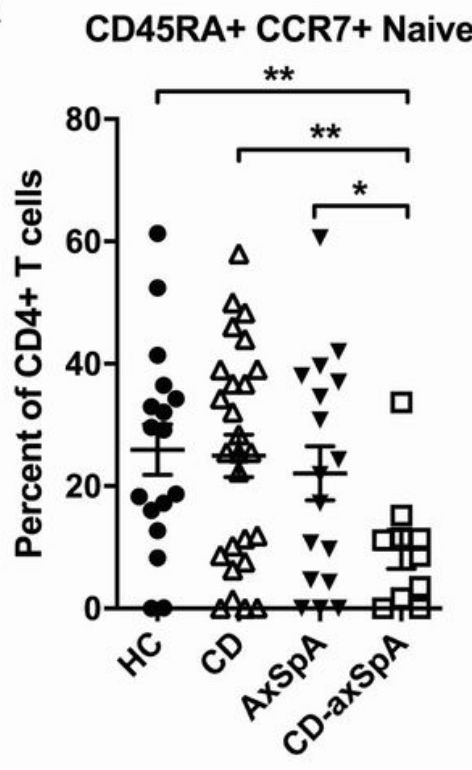

d

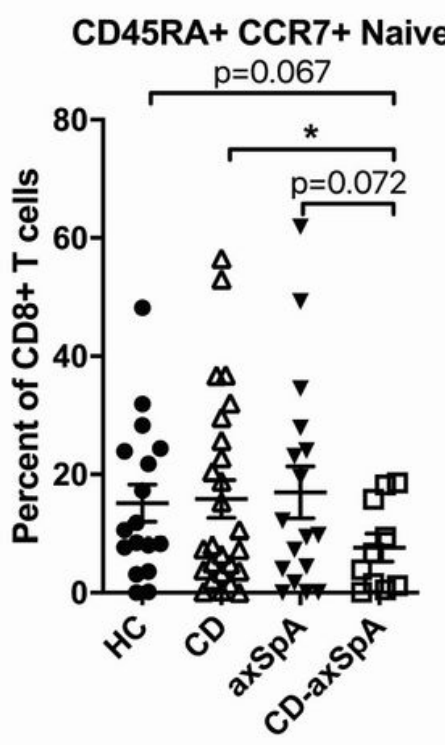

b

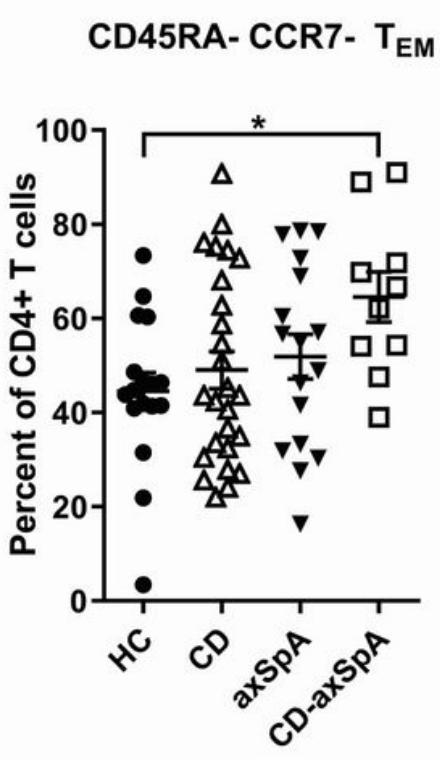

e

PD-1+

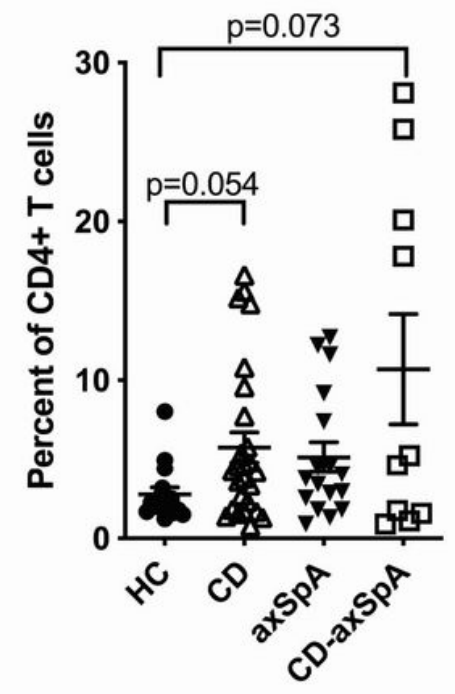

C

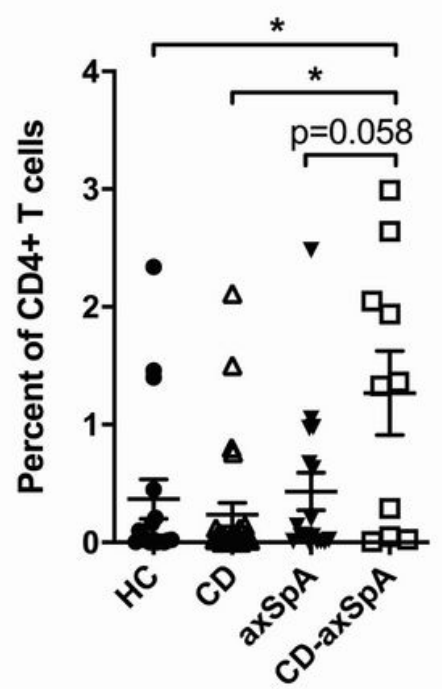

f

PD-1+

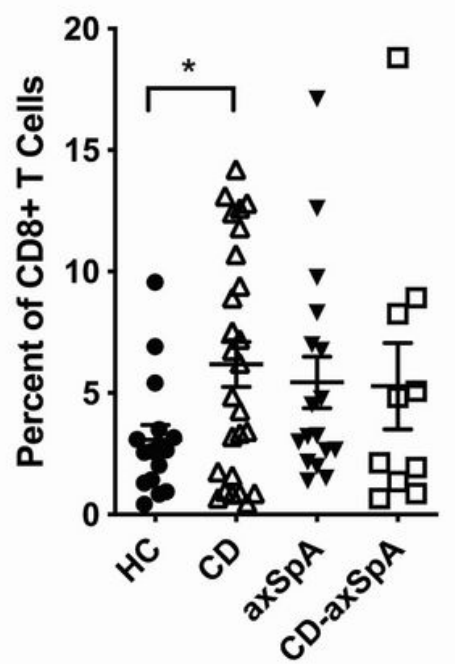

Figure 3

T cells in patients with $\mathrm{CD}$-axSpA are skewed towards a cytotoxic effector memory profile. Singlet, viable lymphocytes gated on CD3+ TCR $\$$ + cells followed by CD4+ and CD8+ per group were evaluated for the percent of (a) CD45RA+ CCR7+ naïve cells versus (b) CD45RA- CCR7- effector memory T cells (TEM) and 
(c) GZMB+ TEM within the CD4+ population. (d) Of the CD8+ T cells, the percent naïve CD45RA+ CCR7+ cells are shown. The percent of PD-1+ cells in the (e) CD4+ and (f) CD8+ populations are shown. Data are shown as individual subjects (dots) with bars as the mean \pm SEM. ${ }^{*}, \mathrm{P}<=0.05$ as determined by MannWhitney for non-parametric data and t-test for parametric data as determined by D'Augostino and Shapiro-Wilk tests.

a

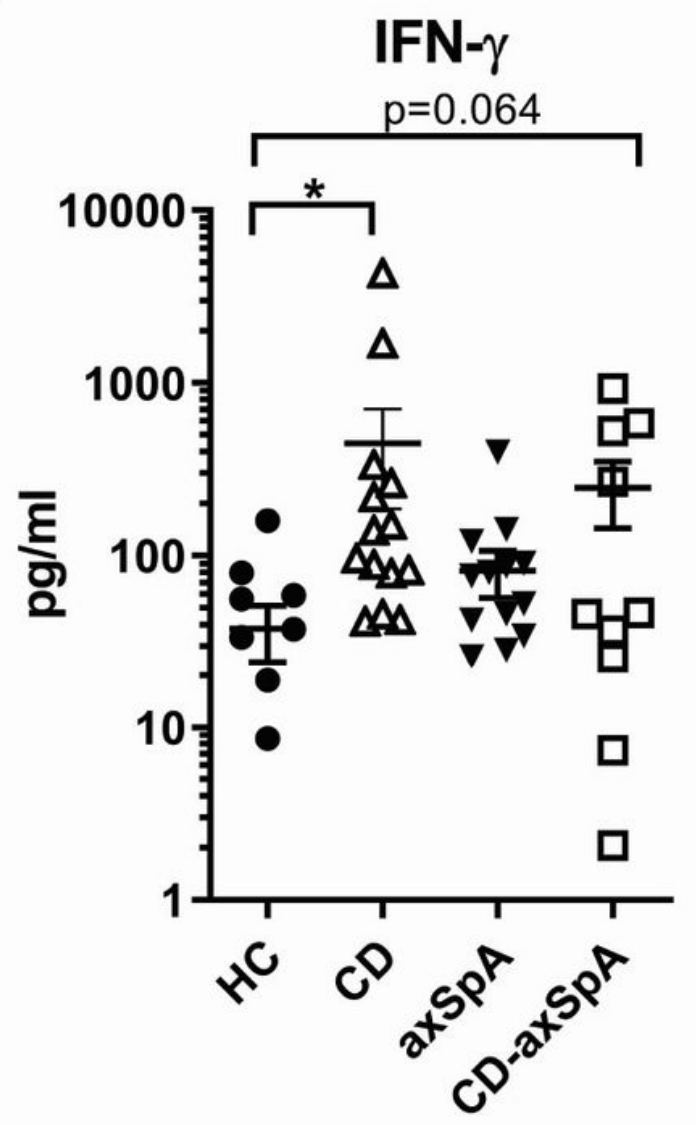

b

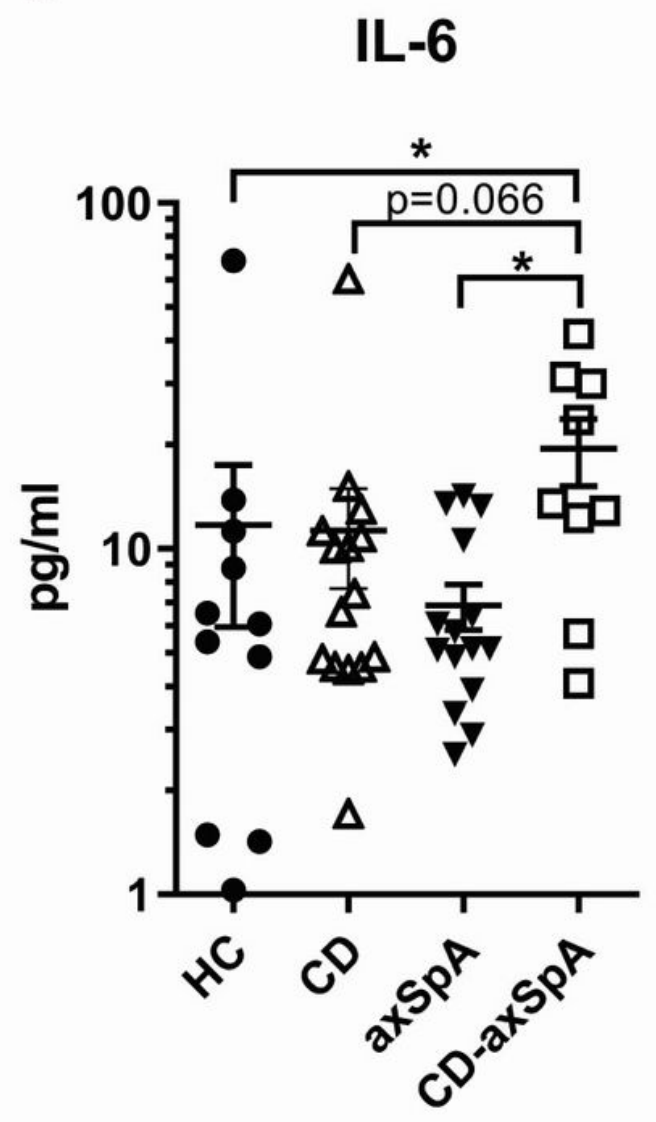

Figure 4

Plasma IFN- $₫$ and IL- 6 are elevated in subjects with CD-axSpA. Plasma from our cohort was evaluated for circulating cytokines by a multiplex ELISA. Symbols represent individual values of (a) IFN- $\square$ and (b) IL-6 while bars are the group mean \pm SEM in $\mathrm{pg} / \mathrm{ml}$. * denotes $\mathrm{P}<0.05$ determined by Mann-Whitney tests. Values below the limit of detection for the assay were not plotted.

\section{Supplementary Files}

This is a list of supplementary files associated with this preprint. Click to download.

- Supplementarymaterials.pdf 